\title{
Image Analysis and DC Conductivity Measurement for the Evaluation of Carbon Nanotube Distribution in Cement Matrix
}

\author{
I. W. Nam, and H. K. Lee*
}

(Received September 22, 2015, Accepted November 26, 2015, Published online December 18, 2015)

\begin{abstract}
The present work proposes a new image analysis method for the evaluation of the multi-walled carbon nanotube (MWNT) distribution in a cement matrix. In this method, white cement was used instead of ordinary Portland cement with MWNT in an effort to differentiate MWNT from the cement matrix. In addition, MWNT-embedded cement composites were fabricated under different flows of fresh composite mixtures, incorporating a constant MWNT content $(0.6 \mathrm{wt} \%)$ to verify correlation between the MWNT distribution and flow. The image analysis demonstrated that the MWNT distribution was significantly enhanced in the composites fabricated under a low flow condition, and DC conductivity results revealed the dramatic increase in the conductivity of the composites fabricated under the same condition, which supported the image analysis results. The composites were also prepared under the low flow condition $(114 \mathrm{~mm}<$ flow $<126 \mathrm{~mm})$, incorporating various MWNT contents. The image analysis of the composites revealed an increase in the planar occupation ratio of MWNT, and DC conductivity results exhibited dramatic increase in the conductivity (percolation phenomena) as the MWNT content increased. The image analysis and DC conductivity results indicated that fabrication of the composites under the low flow condition was an effective way to enhance the MWNT distribution.
\end{abstract}

Keywords: cement composites, carbon nanotubes, distribution evaluation, image analysis, electrical conductivity, fluidity.

\section{Introduction}

The electrical and mechanical properties of cement composites with conductive fibers are significantly influenced by the fiber distribution (Sorensen et al. 2014; Liu et al. 2011; Lee et al. 2009). The electrical properties can be enhanced by the formation of conductive fiber networks. The mechanical properties can also be improved by the effect of fibers bridging micro-cracks, whereas they deteriorated in the base of a non-uniform distribution of fibers (Sorensen et al. 2014; Liu et al. 2011). Accordingly, evaluation of the fiber distribution is important in understanding its influence on the physical properties of the composite materials and making full use of fibers (Liu et al. 2011).

Evaluation methods for the fiber distribution can be classified into two categories - image analyses and electrical property analyses. An image analysis entails acquisition of images via a microscope (optical microscope, scanning electron microscope (SEM), etc.) or a transmission X-ray and image processing and analysis. Lee et al. (2009) and Kang et al. (2011) obtained cross sectional images of composite materials via an

Department of Civil and Environmental Engineering, Korea Advanced Institute of Science and Technology, Daejeon 34141, South Korea.

*Corresponding Author; E-mail: haengki@kaist.ac.kr Copyright $\odot$ The Author(s) 2015. This article is published with open access at Springerlink.com optical microscope (Lee et al. 2009; Kang et al. 2011). Lee et al. (2002) and Liu et al. (2011) obtained images via digital cameras (Lee et al. 2002; Liu et al. 2011). Redon et al. (1999) used X-ray photography and Fan et al. (2000) used a SEM to capture fiber distribution images (Redon et al. 1999; Fan et al. 2000). On the other hand, the fiber distribution state can also be evaluated by the DC conductivity or AC impedance. Li et al. (2007) analyzed MWNT distribution states using the DC conductivity and Ozyurt et al. (2006) evaluated the degree of carbon fiber clumping and fiber orientation by AC impedance spectroscopy (Li et al. 2007; Ozyurt et al. 2006). In the case of the electrical property analysis method, it is only applicable to conductive fiber-incorporated composite materials.

The image analysis is a more direct and convincing approach in that actual fiber distribution states in the composite materials can be evaluated. The electrical property analysis method is an indirect approach for evaluation of the fiber distribution, but sometimes it is affected by other parameters (matrix type, moisture content in matrix, etc.), possibly leading to incorrect electrical property results. Accordingly, the image analysis plays an important role in the evaluation of fiber distributions and it is a critical technique that should be carried out along with an electrical property analysis (Li et al. 2007; Ozyurt et al. 2006).

Uncovered in 1991, carbon nanotube (CNT) has gained great attention from researchers attributable to its remarkable physical properties (Li et al. 2007; Kim et al. 2014). From 2010s, CNT has been used as a nano-scale fiber in cementbased composites in an effort to enhance physical properties 
of the composites (Kim et al. 2014). Various approaches have been explored to evaluate CNT distributions in the literature. SEM observation has been the most common approach to study the CNT distribution state, carried out in tandem with an electrical property analysis (Konsta-Gdoutos et al. 2010). However, the SEM observations may vary depending on observation spots in the same sample since it is mostly conducted with high magnification levels (up to 3000-fold). Accordingly, the CNT distribution should be observed and evaluated at low magnification levels and the evaluation results should be compared with electrical properties of the CNT-embedded composites.

In the present work, a novel image analysis method to evaluate the multi-walled carbon nanotube (MWNT) distribution in cement-based composites is proposed. In this approach, white cement was used instead of ordinary Portland cement (OPC) with MWNT in an effort to differentiate MWNT from the cement matrix. MWNT distribution images in the cement matrix materials were acquired by using an optical microscope in conjunction with image processing tools. The MWNT distribution was quantitatively assessed in terms of the planar occupation ratio of MWNT. In preparation of specimens, a novel method was adopted for dispersion of CNT in cement. This method was proposed on the basis of experimental attempts previously conducted by the authors. It was suggested that the distribution of CNT can be enhanced by means of lowering the fluidity of CNT/ cement mixture at fresh state. It is notable that the suggested method does not require sonication technique, acid treatment, etc., which were widely demonstrated in the literature. To verify correlation between the MWNT distribution and flow of the mixtures, MWNT-embedded cement composites were fabricated under different flows, incorporating a constant MWNT content $(0.6 \mathrm{wt} \%)$. The image analysis demonstrated that the MWNT distribution was significantly enhanced in the composites fabricated under a low flow condition, and DC conductivity measurement results revealed the dramatic increase in the conductivity of the composites fabricated under the low flow condition, which supported the image analysis results. The composites were also prepared under the low flow condition (114 $\mathrm{mm}<$ flow $<126 \mathrm{~mm}$ ), incorporating various MWNT contents. The image analysis and DC conductivity results demonstrated remarkable enhancement in the planar occupation ratio of MWNT and the conductivity, respectively, which indicated that fabrication of the composites under the low flow condition was an effective way to enhance the MWNT distribution.

\section{Materials}

MWNT, Portland cement, nylon fiber, super-plasticizer, tap water, and silica fume (SF) were used in the present work. MWNT produced through the chemical vapor deposition (CVD) growth method, a proprietary product of Hyosung Inc. (M1111), was used (Nam et al. 2012). Their purity, diameter, and aspect ratio were $96.2 \%, 12.29 \pm 2.18 \mathrm{~nm}$, and 930 (aspect ratio was approximate value), respectively. Type I ordinary Portland cement was used in the present work. SF, a proprietary product of Elkem Inc. (EMS-970 D), contained over $90 \%$ silicon oxide $(\mathrm{SiO})$ and $80 \mathrm{wt} \%$ of its primary particles have a diameter greater than $5 \mu \mathrm{m}$. White cement was used instead of OPC when image analysis samples were fabricated. Nylon fiber, a proprietary product of Nycon fibers Inc. (NYMAX), was used in an effort to prevent cracks that occur due to shrinkage while cement matrix materials cured. Their diameter and length were $23-36 \mu \mathrm{m}$ and $3 \mathrm{~mm}$, respectively. A poly-carboxylic acid-based super-plasticizer (SP), a proprietary product of BASF Pozzolith Ltd., (Rheobuild SP8HU), was utilized in an effort to improve the workability of the fresh cement matrix materials. The true specific gravity of MWNT, Portland cement, SP, tap water, and SF was 1.32, $3.15,1.07,1$, and 2.1 , respectively.

\section{Methods}

\subsection{Image Analysis of MWNT Distribution in MWNT-Embedded Cement Composites \\ 3.1.1 Specimen Preparation and Image Acquisition for Image Analysis}

A novel image analysis approach was proposed in an effort to evaluate the MWNT distribution in cement matrix materials. In order to distinguish MWNT with black color from OPC, which also shows a fairly dark color, white cement was used in place of OPC when composite specimens were fabricated for the image analysis. In addition to using white cement instead of OPC, the incorporation of nylon fiber was omitted in the preparation of the samples for the image analysis since crack control was unnecessary. The constituent materials (white cement, MWNT, water, and SP) were weighed according to mixing ratios and placed together in a steel bowl.

Table 1 shows the mix proportions of composites fabricated with different $\mathrm{W} / \mathrm{C}$ values, which will be dealt in Sect. 4.1.1. The mix proportions for composites fabricated under a controlled low flow condition (from 114 to $126 \mathrm{~mm}$ ) with various MWNT contents are presented in Table 2, which will be dealt in Sect. 4.2.1. The weighed materials were thoroughly mixed for 20 min by an electric hand mixer. The electric hand mixer consisted of two curl shaped-beaters and their rotation rate was approximately $200 \mathrm{rpm}$. The fresh mixtures were poured into plastic molds that were designed in accordance with the sample size. The sample size was $10 \times 10 \times 80\left(\mathrm{~mm}^{3}\right)$ for the image analysis. After pouring the fresh mixtures in the molds, they were covered with a plastic plate (or wrapping film) in an effort to avoid moisture loss of the samples and cured for $24-48 \mathrm{~h}$ at room temperature $\left(18-20{ }^{\circ} \mathrm{C}\right)$ under a humid condition (40-50\%), and then detached from the molds.

In an effort to produce a fracture surface, a cutting knife was used to make a cutting guide line with depth of approximately $1 \mathrm{~mm}$ at the center of the sample. The sample was split into two parts applying manual force by hand to the cutting guide. Each split part was sliced by means of a hand saw at $3 \mathrm{~mm}$ from the fracture surface. As a result, a 
Table 1 Constituent materials and their mix proportions of the MWNT-embedded cement composites fabricated under different flow.

\begin{tabular}{|c|c|c|c|c|c|c|c|}
\hline \multirow[t]{2}{*}{ Denotations } & \multicolumn{6}{|c|}{ Mix proportions (g) } & \multirow[t]{2}{*}{ MWNT (vol\%) } \\
\hline & Water & Cement & $\mathrm{SF}$ & $\mathrm{SP}$ & Nylon fiber & MWNT & \\
\hline W26-M06 & 26 & \multirow[t]{5}{*}{100} & \multirow[t]{5}{*}{20} & \multirow[t]{5}{*}{1.6} & \multirow[t]{5}{*}{0 (omitted) } & \multirow[t]{5}{*}{0.6} & 0.66 \\
\hline W30-M06 & 30 & & & & & & 0.62 \\
\hline W34-M06 & 34 & & & & & & 0.59 \\
\hline W38-M06 & 38 & & & & & & 0.56 \\
\hline W42-M06 & 42 & & & & & & 0.53 \\
\hline
\end{tabular}

Table 2 Constituent materials and their mix proportions of the composites fabricated under the low flow condition $(114 \mathrm{~mm}<$ flow $<126 \mathrm{~mm})$.

\begin{tabular}{|c|c|c|c|c|c|c|c|c|}
\hline \multicolumn{2}{|c|}{ Denotations } & \multicolumn{6}{|c|}{ Mix proportions (g) } & \multirow[t]{3}{*}{ Flow $(\mathrm{mm})$} \\
\hline \multirow[t]{2}{*}{ Group } & \multirow[t]{2}{*}{ Type } & \multirow[t]{2}{*}{ Water } & \multirow[t]{2}{*}{ Cement } & \multicolumn{2}{|c|}{ MWNT } & \multirow[t]{2}{*}{ SP } & \multirow[t]{2}{*}{ Nylon fiber } & \\
\hline & & & & $\begin{array}{l}\mathrm{Wt} \% \text { by } \\
\text { cement }\end{array}$ & Vol\% & & & \\
\hline \multirow[t]{5}{*}{ LF-M } & LF-M0 & 25 & \multirow[t]{5}{*}{100} & 0 & 0 & 0.6 & \multirow[t]{5}{*}{0.2} & 160 \\
\hline & LF-M0.3 & 25 & & 0.3 & 0.39 & 1.0 & & 126 \\
\hline & LF-M0.6 & 25 & & 0.6 & 0.76 & 3 & & 125 \\
\hline & LF-M1.0 & 30 & & 1.0 & 1.14 & 4.54 & & 120 \\
\hline & LF-M1.5 & 36 & & 1.5 & 1.56 & 4.4 & & 114 \\
\hline
\end{tabular}

specimen with size of $10 \times 10 \times 3 \mathrm{~mm}^{3}$ was prepared from the original sample.

A magnification to cover MWNT agglomerates whose diameter ranged from 3 to $200 \mu \mathrm{m}$ was selected by a trial and error process and determined as 50-fold. An auto stage optical microscope (Olympus MX51) was used so that visible light could be used to distinguish MWNT (black) from the cement matrix (white). The microscope system illuminates the specimen with uniform brightness and transfers the observation image to image processing software, DotSlide. Since the optical focus at each local spot of the fractured specimen changes due to unevenness of the specimen surface, the microscope system captured observation images at every $3 \mu \mathrm{m}$ along the out of plane direction from the deepest level to the highest level of the fractured surface. By using the image processing software, focused local spots in the captured images were collected and combined to form a single image that is in focus overall. Two images of each specimen were gained through the aforementioned procedure.

\subsubsection{Image Processing and Analysis Procedures}

In microscopic images, the black amorphous agglomerates indicated MWNTs. The contrast of white cement and MWNT was seen remarkably well by the naked eye. By image processing and analysis with the commercial software, the MWNT distribution can be quantitatively evaluated. Stepwise tasks were carried out in the following order (illustrations for each step can be found in Fig. 1) with the fractured specimens.
(1) Image acquisition by microscope in collaboration with a digital camera

(2) Capture areas that are optically well focused and assemble them so a well-focused image can be completed (conducted by DotSlide)

(3) Convert the image to a black and white image by thresholding

(4) Reverse the black and white image

(5) Convert the black and white image to a binary image

(6) Acquire data (area) of agglomerate regions

Steps (1) to (2) were conducted by the microscope system and the remaining steps were conducted using a MATLAB Image processing tool box. Automatic brightness control was applied in step (1) by the microscope system. The effect of change of brightness level on the image thresholding was considered a minor factor and hence was ignored in the present work. Otsu (1979)'s automatic thresholding method was used in the thresholding process of step (3) (Otsu 1979). The gray-level scale, which is determined between 0 and 1 , was used with a $15 \%$ reduction (gray-level scale $\times 0.85$ ) since the brightness of MWNT was considered high when compared with the original image. After the reversion process (4), bright parts indicated MWNT. Accordingly, the planar occupation area of the bright parts expressed as 1 in the corresponding binary image, was summed. The planar occupation ratio of bright parts can be calculated by dividing the sum total of the bright parts by the total area of the corresponding image. The planar occupation ratio of MWNT 


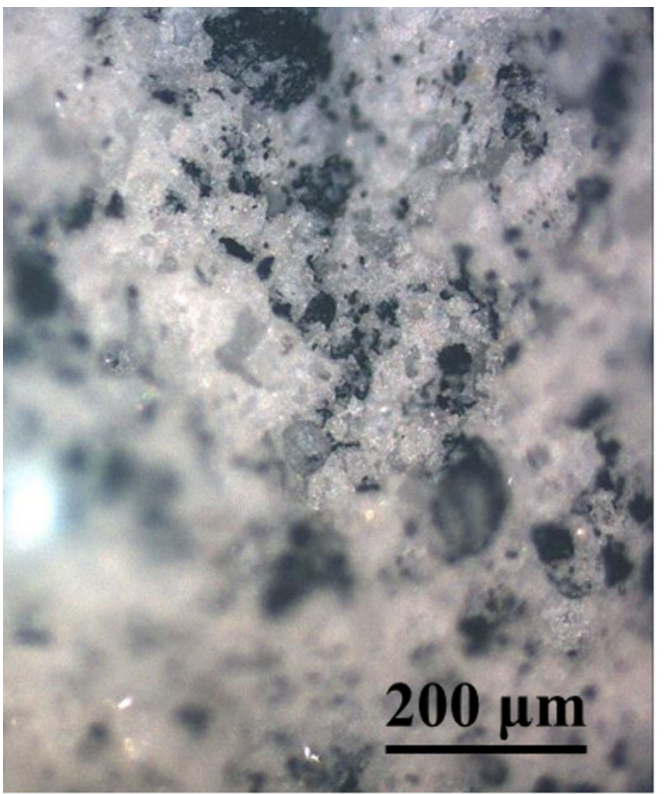

(a)

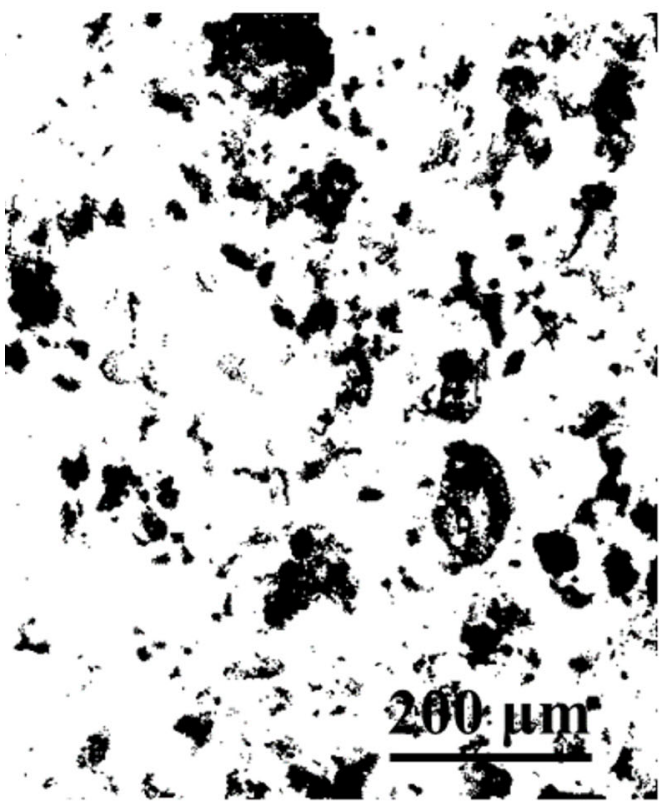

(c)

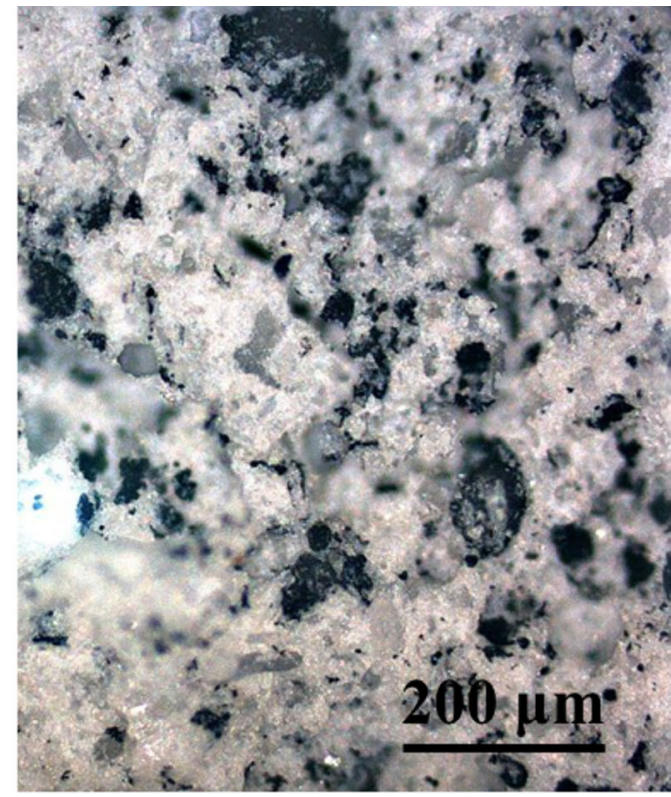

(b)

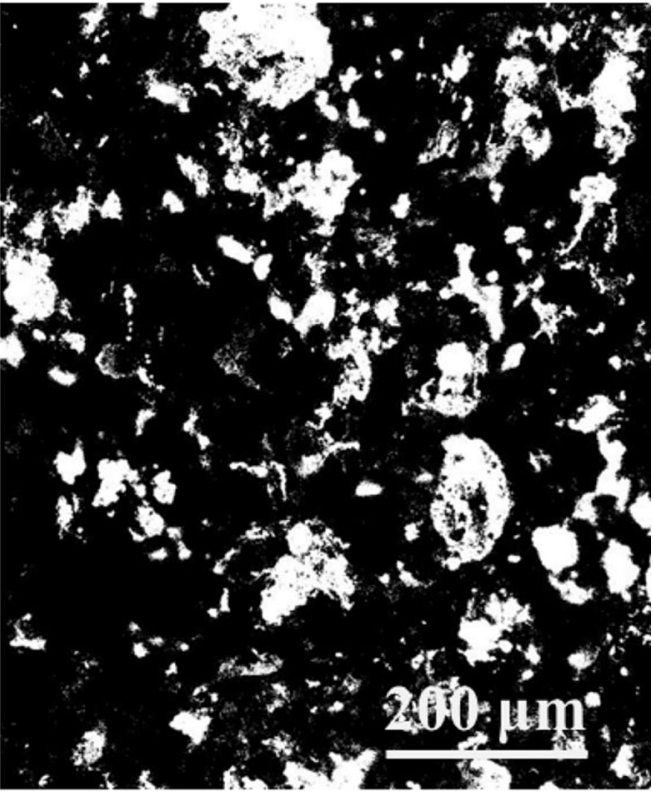

(d) and (e)

Fig. 1 Image processing procedures (image size: $295 \times 355$ pixel): a Image acquisition, b gathering of areas that are well optically focused and assemble them together, c conversion of the image to a black and white image by thresholding, d reversal of the image, and e conversion of the image to a binary image.

was calculated from each of two images and an average value was determined from the ratios.

\subsection{Conductivity of the MWNT-Embedded Cement Composites}

\subsubsection{Specimen Preparation for DC Conductivity}

\section{Measurement}

The preparation procedures of samples for DC conductivity measurement followed the specimen preparation procedures for the image analysis in Sect. 3.1.1. However, OPC was used as cement material in this experiment and nylon fiber was used. The constituent materials of the composites were prepared in accordance with mix proportions. In particular, the mix proportions for composites prepared with different flows and constant MWNT content are presented in Table 3, and DC conductivity of the composites will be dealt in Sect. 4.1.2. On the other hand, Tables 2 and 4 show the mix proportions of composites fabricated under the low flow condition and a high flow condition (over $250 \mathrm{~mm}$ ), which will be dealt in Sect. 4.2.2 in terms of DC conductivity. The constituent materials were weighed according to the mix proportions and mixed by using the electric hand mixer. The resultant fresh mixture was decanted into plastic molds designed with the size $25 \times 25 \times 25\left(\mathrm{~mm}^{3}\right)$. After undergoing 1 day's curing under conditions explained in Sect. 3.1.1, the samples were separated from the molds. In an effort to gain mechanical strength 
Table 3 The constituent materials and their weight content ratios of the composites fabricated under different flow in an effort to understand change of the MWNT distribution by examining the DC conductivity of the composites.

\begin{tabular}{|c|c|c|c|c|c|c|c|}
\hline \multirow[t]{2}{*}{ Denotations } & \multicolumn{7}{|c|}{ Mix proportions (g) } \\
\hline & Water & Cement & SF & SP & Nylon fiber & MWNT & Flow (mm) \\
\hline M06-W26 & 26 & \multirow[t]{11}{*}{100} & \multirow[t]{11}{*}{20} & \multirow[t]{6}{*}{1.6} & \multirow[t]{11}{*}{0.2} & \multirow[t]{11}{*}{0.6} & 102 \\
\hline M06-W30 & 30 & & & & & & 127 \\
\hline M06-W34 & 34 & & & & & & 151 \\
\hline M06-W38 & 38 & & & & & & 211 \\
\hline M06-W40 & 40 & & & & & & 250 \\
\hline M06-W42 & 42 & & & & & & $>250$ \\
\hline M06-SP0 & \multirow[t]{5}{*}{40} & & & 0 & & & 130 \\
\hline M06-SP04 & & & & 0.4 & & & 119 \\
\hline M06-SP16 & & & & 1.6 & & & 250 \\
\hline M06-SP32 & & & & 3.2 & & & $>250$ \\
\hline M06-SP64 & & & & 6.4 & & & $>250$ \\
\hline
\end{tabular}

Table 4 The constituent materials and their mix proportions of the composites fabricated under the high flow condition (flow $>250 \mathrm{~mm}$ ).

\begin{tabular}{|c|c|c|c|c|c|c|c|}
\hline \multicolumn{2}{|c|}{ Denotations } & \multicolumn{6}{|c|}{ Mix proportions $(\mathrm{g})$} \\
\hline \multirow[t]{2}{*}{ Group } & \multirow[t]{2}{*}{ Type } & \multirow[t]{2}{*}{ Water } & \multirow[t]{2}{*}{ Cement } & \multicolumn{2}{|c|}{ MWNT } & \multirow[t]{2}{*}{ SP } & \multirow[t]{2}{*}{ Nylon fiber } \\
\hline & & & & $\mathrm{Wt} \%$ by cement & Vol\% & & \\
\hline \multirow[t]{4}{*}{ HF-M } & HF-M0 & \multirow[t]{4}{*}{40} & \multirow[t]{4}{*}{100} & \begin{tabular}{|l|}
0 \\
0
\end{tabular} & 0 & \multirow[t]{4}{*}{1.6} & \multirow[t]{4}{*}{0.2} \\
\hline & HF-M0.3 & & & 0.3 & 0.31 & & \\
\hline & HF-M0.6 & & & 0.6 & 0.62 & & \\
\hline & HF-M1.0 & & & 1.0 & 1.02 & & \\
\hline
\end{tabular}

to prevent unexpected damages in the samples, they were submerged in water during 7 days.

\subsubsection{Measurement Method}

Li et al. (2007) and Xie et al. (1996) revealed that the CNT distribution in CNT-added composite materials can be assessed by examining the DC conductivity of the materials (Li et al. 2007; Xie et al. 1996). Once SEM or transmission electron microscopy (TEM) reveal that CNTs are homogeneously dispersed in the composite materials, DC conductivity evaluation can be used in an effort to identify the percolation network, which is a large cluster network spanning from one side to the opposite side without disconnection in the composite materials (Stauffer and Aharony 1994).

The measurement method of DC conductivity in the present work complied with a standard method presented in SEMI MF43 (2005). As electrodes for the measurement, a pair of copper plates having dimensions of $10 \times 35 \times 0.5$ $\left(\mathrm{mm}^{3}\right)$ was inserted in the center part of the sample with a spacing of $8 \mathrm{~mm}$ as shown in Fig. 1 of Nam et al. (2015). The copper electrodes were embedded when the composite mixtures were still fresh. As another pair of electrodes, both sides of the samples were coated with silver paste as shown in Fig. 1 of Nam et al. (2015). A power supply equipment (Agilent E3642A) generated DC current, and it was passed through the silver paste electrodes. Due to the supply of current in resistors, which are the MWNT-embedded cement composites, potential difference in the composites was created, and it was measured by a digital multimeter (Agilent 34410A) connected with the copper plates. The supplied current was automatically controlled by the power supplier with a limitation up to $0.2 \mathrm{~A}$ and the resultant voltage was produced with a limitation up to $20 \mathrm{~V}$. Figure 2 illustrates the measurement method for DC conductivity.

The resistance of the composites was calculated on the basis of Ohm's law. For the determination of DC conductivity, the calculated resistance value $(R)$, cross sectional area of the electrode in contact with the composites $(A)$, and the interval of the electrodes $(L)$ were plugged into a following equation (Xie et al. 1996; Vance et al. 2014)

$$
\sigma=\frac{1}{\rho}=\frac{L}{R \times A}=\frac{0.8 \mathrm{~cm}}{R \times(2.5 \mathrm{~cm} \times 1 \mathrm{~cm})}(\mathrm{S} / \mathrm{cm})
$$

\subsection{Flow of the Composite Mixtures}

The flow of fresh mixture of the composites was tested on the basis of ASTM C1437 (ASTM International 2013). In 


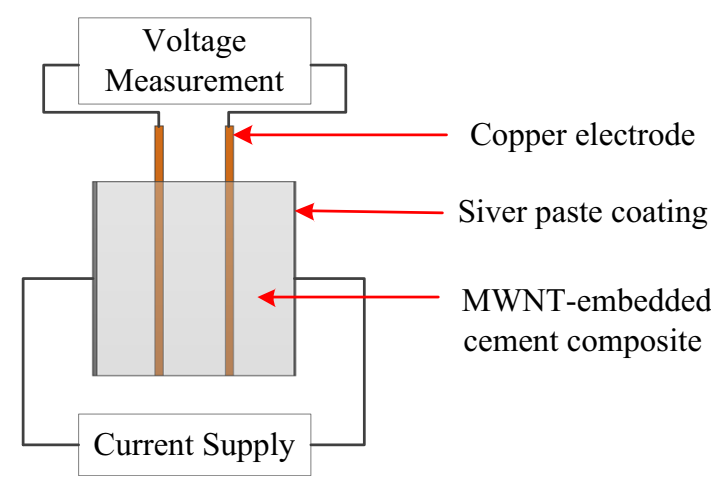

Fig. 2 A schematic illustration of the DC conductivity measurement for the composites.

addition, the flow was determined from an average of mixture's diameter after dropping the flow table.

\section{Results and Discussion}

\subsection{MWNT Distribution in Composites Fabricated Under Different Flows}

4.1.1 Image Analysis of MWNT Distribution State in the Composites Fabricated Under Different Flows

Through preliminary tests on MWNT-embedded cement composites, it is found that the flow of the fresh mixture may influence the MWNT distribution. Accordingly, MWNTembedded cement composites fabricated with different W/C values were prepared and the MWNT distribution state of the composites was evaluated. The constituent materials are listed in Table 1 . The mixing ratio of MWNT was $0.6 \mathrm{wt} \%$ in order to provide a sufficient amount of MWNT. $0.6 \mathrm{wt} \%$ of MWNT exceeded the percolation threshold as reported in the literature (Nam et al. 2015). Accordingly, the distribution of MWNT was expected to be pronouncedly visible. The volumetric fractions (vol\%) of MWNT were also provided in the table. In determination procedures of volumetric fractions of MWNT, volume of each constituent material had to be calculated by using true specific gravity of the materials shown in the Sect. 2, then volume ratio of MWNT to total volume of the mixture was obtained. $20 \%$ (by cement weight) SF was added in the mixtures under the consideration that SF can improve the MWNT distribution (Nam et al. 2012). The white cement was substituted for OPC and the incorporation of nylon fiber was omitted, as addressed in Sect. 3.1.1. The specimens were prepared and images of fractured surfaces of the specimens were obtained according to the method described in Sect. 3.1.2.

Figure 3 presents processed images that were obtained from the fabricated specimens. In the processed images, the total area of the MWNT agglomerates appeared to decrease with an increase of the flow (or W/C ratio). Moreover, MWNT clumps, which are excessively entangled-MWNT agglomerates, were generated as the flow increased. Based on these observations, it can be surmised that MWNT agglomerates disentangled as the flow (or W/C ratio) decreased.
In an effort to express the change of the MWNT distribution states in a quantitative manner, the proportion of MWNT agglomerates, indicated by white color, to the total area of the image, was calculated by the MATLAB image processing tool box. This proportion is designated as a $q$ value. The $q$ value refers to the planar occupation ratio of MWNT on a fractured surface of the composite.

Figure 4 shows an increase of the $q$ value as the $\mathrm{W} / \mathrm{C}$ ratio of the cement composites decreases. The $q$ value doubled due to a $16 \%$ reduction of the $\mathrm{W} / \mathrm{C}$ ratio. The increase of the $q$ value was attributed to disentanglement of the MWNT agglomerates. The disentanglement of the MWNT agglomerates can be explained by variation of the microstructure of the fresh mixture. Figure 5a shows the microstructure of the fresh mixture of the MWNT-embedded cement composite prepared under a high flow condition. The distance between unhydrated cements increased as the $\mathrm{W} / \mathrm{C}$ ratio (or $\mathrm{SP} / \mathrm{C}$ ratio, i.e. SP versus cement ratio) increased (Daimon and Roy 1979). When the interspace among the unhydrated cements increased, highly entangled MWNTs floated in the free water. This is ascribed to hydrophobic characteristics of MWNT. As a result, the images obtained from the W38M06 and W42-M06 samples showed the highly entangled MWNTs, as presented in the Fig. 3. On the contrary, the microstructure of the fresh mixture of the composites can be changed if the flow of the mixture is decreased. The distance between unhydrated cements in the composites prepared under a low flow condition was narrowed, as shown in Fig. 5b. If the interspace is narrowed, the MWNT agglomerates can be disentangled during the mixing process. This is attributable to the characteristic that the size of the MWNT agglomerates can be restricted by the size of the interspace among the unhydrated cements. As a result, the images obtained from the W26-M06 and W30-M06 specimens in the Fig. 3 showed disentangled MWNT agglomerates.

The planar occupation ratio of MWNT, $q$, was increased twofold with decrease of W/C ratio from 42 to $26 \%$ as shown in the image analysis result of Fig. 4. However, electrical conductivity of the composites is expected to increase more than hundreds of times as the $\mathrm{W} / \mathrm{C}$ ratio decreases. This stems from that disconnected CNTs can be electrically connected attributable to the enhancement of MWNT distribution. Accordingly, it can be said that the twofold greater $q$ value can lead to a dramatic increase in the electrical conductivity. This will be dealt in the electrical property characterization section.

\subsubsection{Conductivity of the Composites Fabricated Under Different Flows}

The image analysis of the MWNT distribution state of the MWNT-embedded cement composites fabricated under different flows (or W/C) studied in Sect. 4.1.1 showed that the MWNT distribution improved as the flow of the composite decreased. In the present section, the DC conductivity of the composites was examined in an effort to understand the change of the MWNT distribution in the composites and also to verify whether it supported the image analysis results. The constituent materials and respective weight content ratios of 


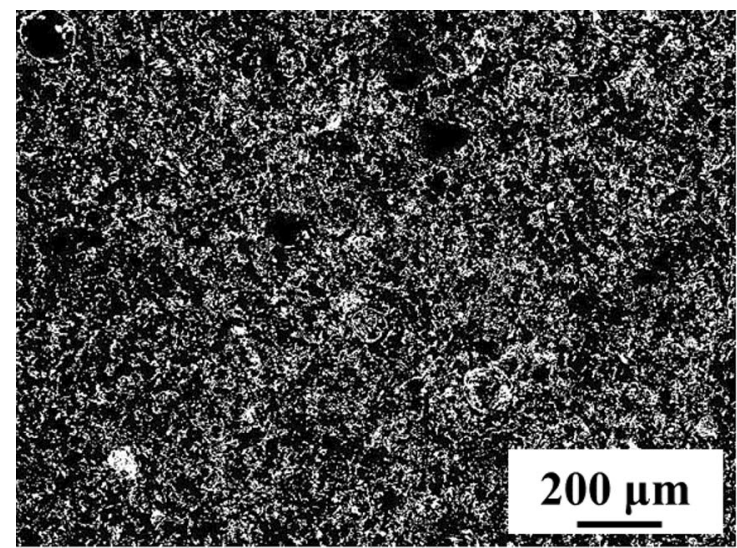

(a) W26-M06

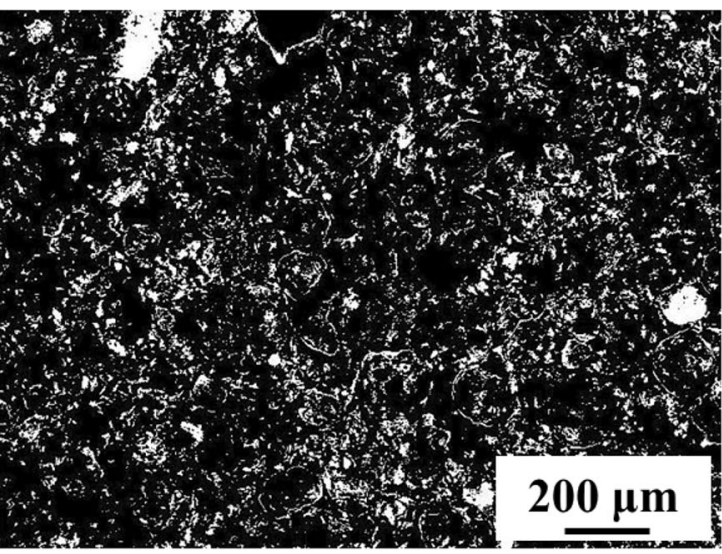

(c) W38-M06

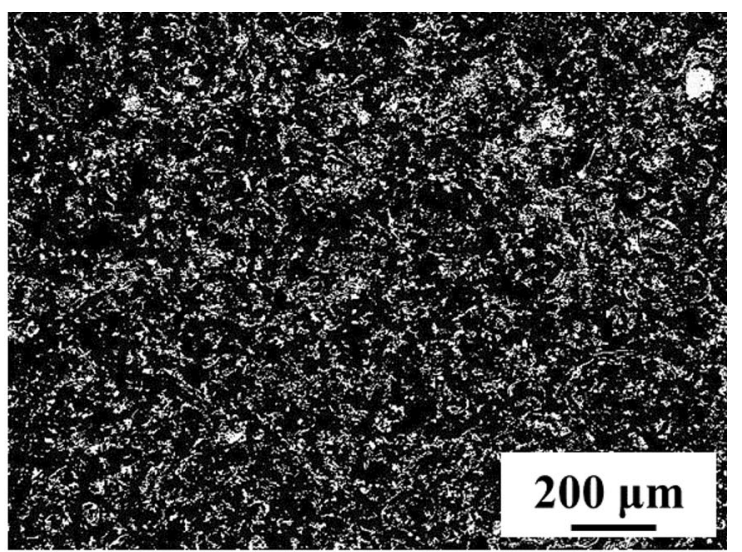

(b) W30-M06

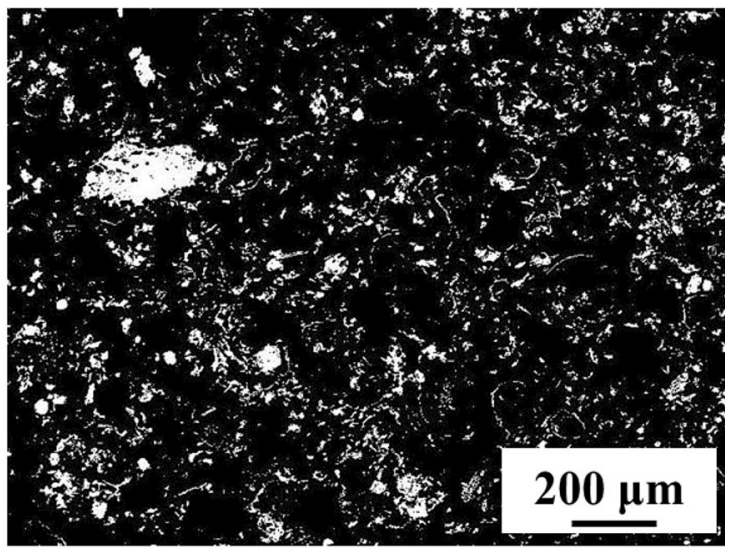

(d) W42-M06

Fig. 3 The fractured surface of the MWNT-embedded cement composites fabricated under different flow (W/C ratio) after having the image thresholding, reversion, and binarization processes (image size: $570 \times 426$ pixel): a W26-M06, b W30-M06, c W38-M06, and d W42-M06.

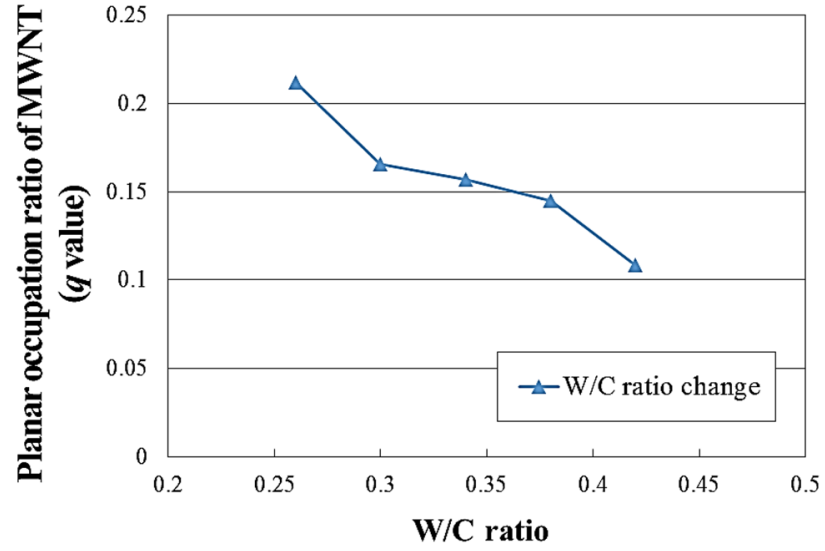

Fig. 4 The variation of the $q$ value, which is the planar occupation of MWNT on the fractured surface of the composites, as a function of W/C ratio.

the composites are given in Table 3. To make specimens with different flows, one group of specimens was fabricated by varying the water content, as described in Sect. 4.1.1, and an additional group of specimens was fabricated by varying the SP content in the present section. The fabrication procedures of the specimens followed descriptions in
Sect. 3.2.1 and the measurement method was described in Sect. 3.2.2.

The variations in the DC conductivity of the specimens are plotted in Fig. 6a, b. The change of the DC conductivity of the specimen group prepared with different water content is shown in the Fig. 6a and the results of the specimen group prepared with different SP content are shown in Fig. 6b. An electrical percolation phenomenon can be observed in the electrical conductivity versus $\mathrm{W} / \mathrm{C}$ ratio plot in the Fig. $6 \mathrm{a}$. This phenomenon indicated that the water content or flow of the mixture is a crucial factor affecting the MWNT distribution of the composites, as found in the image analysis in Sect. 4.1.1. The dramatic increase of electrical conductivity with a decrease of the flow is attributed to disentanglement of the MWNT agglomerates, as shown in the image analysis. In the image analysis, the $q$ value linearly increased with a decrease of the $\mathrm{W} / \mathrm{C}$ ratio but the electrical conductivity exponentially increased with the decrease of the W/C ratio. This was due to the formation of the percolation network, which induced a remarkable increase of the DC conductivity of some orders. The Fig. $6 \mathrm{~b}$, which presents the electrical conductivity versus $\mathrm{SP} / \mathrm{C}$ ratio plot, also shows the electrical percolation phenomenon. This indicates that the flow plays a decisive role in determining the MWNT distribution, 


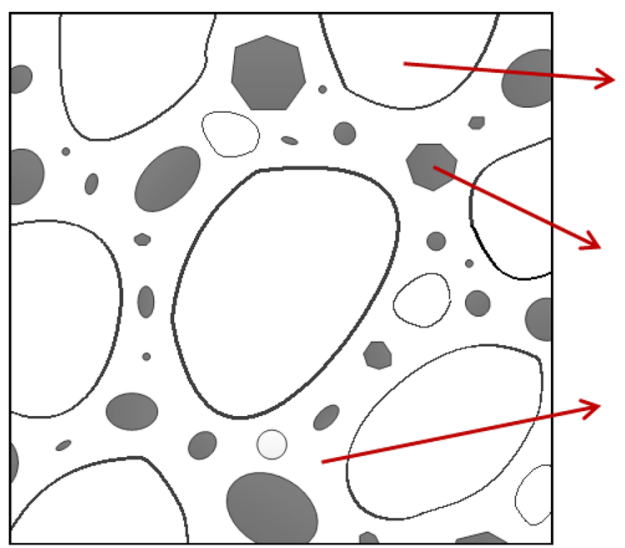

(a)

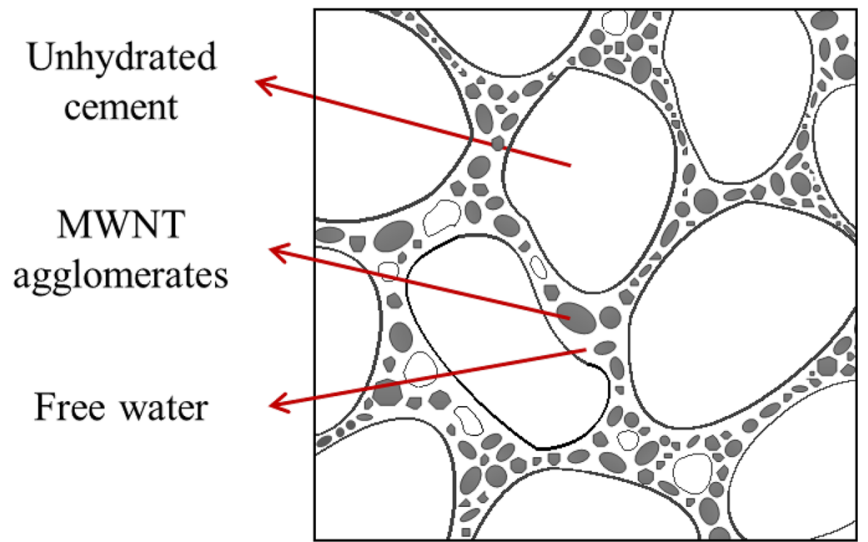

(b)

Fig. 5 Micro structure of fresh mixture of the MWNT-embedded cement composites prepared under the high flow condition (a) and the low flow condition (b).

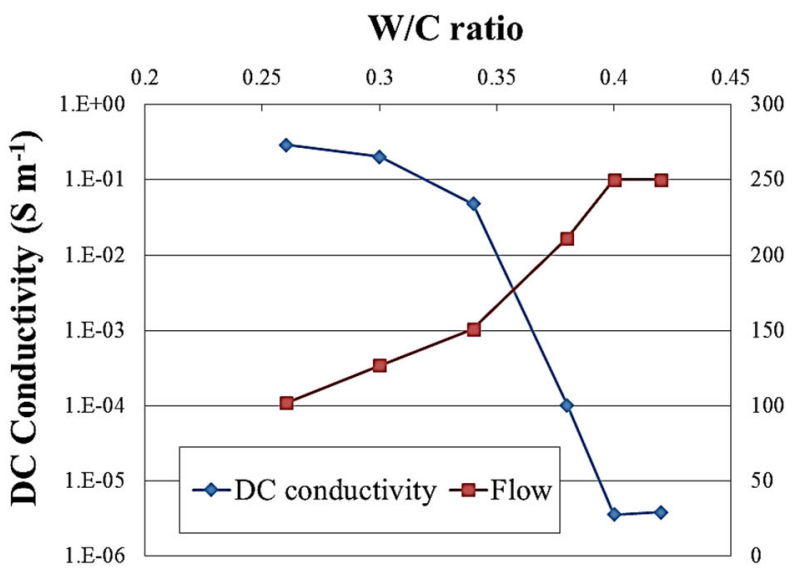

(a)

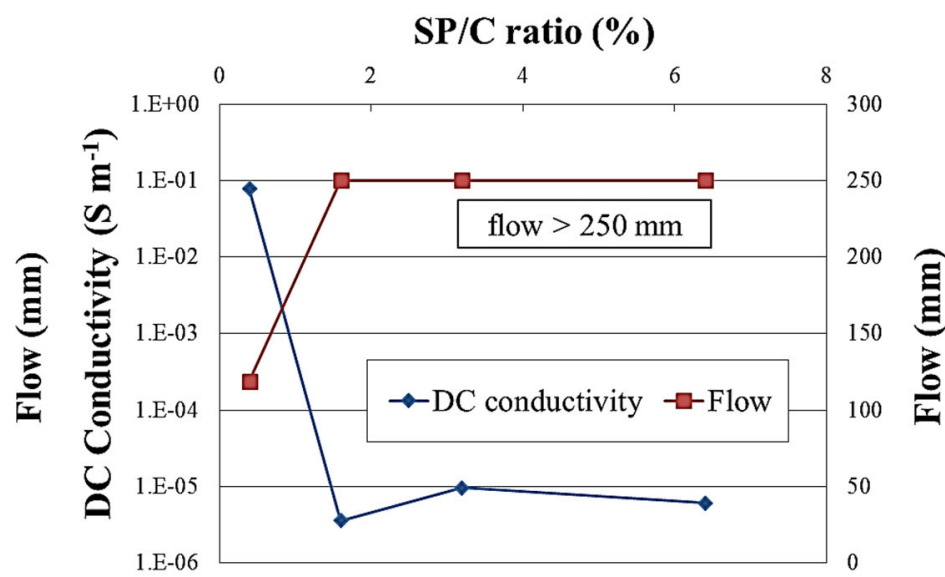

(b)

Fig. 6 The DC conductivity of the MWNT-embedded cement composites fabricated as a function of W/C ratio (a) and SP/C ratio (b).

because water content was constant in the specimen group with different $\mathrm{SP} / \mathrm{C}$ ratio. It is consequently found that the flow of fresh mixture of the composites is an important factor that determines the MWNT distribution state, based on the image analysis and the electrical conductivity evaluation. The improvement of the MWNT distribution with a decrease of the flow is thought to be related to variation of the interspace among unhydrated cement, as discussed in Sect. 4.1.1.

It is worth noting that the electrical conductivity of the M06-W30 was greater than that of the M06-SP04 although the flow of the M06-W30 was higher than that of the M06SP04. This indicates that control of water content can be a more effective way of enhancing the electrical conductivity of the composites.

Therefore, two categories of specimens were prepared in the subsequent experiments. One category included MWNTembedded cement composites fabricated under a high flow condition (flow $>250 \mathrm{~mm}$ ) and another category included composites fabricated under a low flow condition (114 $\mathrm{mm}<$ flow $<126 \mathrm{~mm}$ ) by adjusting the $\mathrm{W} / \mathrm{C}$ ratio and the $\mathrm{SP} / \mathrm{C}$ ratio. To understand the MWNT distribution states, the image analysis and DC conductivity measurement for the specimen groups were carried out.

\subsection{Influence of the Flow on MWNT Distribution in the Composites Incorporating Various MWNT Contents}

\subsubsection{Image Analysis of the MWNT Distribution State in the Composites}

It was found that the flow of fresh mixture was closely related with the MWNT distribution, as observed in Sect. 4.1.2. Accordingly, the MWNT distribution states in MWNT-embedded cement composites with various MWNT contents can be compared by setting the flow of mixtures to be consistently low (flow between 114 and $126 \mathrm{~mm}$ ). Otherwise, the MWNT distribution is so poor that the reliability of the image analysis data obtained from the composites with various MWNT contents can diminish. To prepare composite mixtures with the low flow values, cement composites with various MWNT contents were fabricated by adjusting the content ratios of water and SP. 
The water to cement ratio was controlled with reference to the MWNT content in each batch. This was based on a report that MWNT tends to absorb water within its hollow structure (Striolo et al. 2005). The SP content ratio was also adjusted in consideration of the amount of cement and MWNT, but the final SP content was determined by some trials of SP addition in the mixture and flow test. The constituent materials used in the present work are listed in Table 2 but nylon fiber was not used for the image analysis specimens. The mixing ratio of MWNT ranged from 0 to $1.5 \%$. Figure 7 presents processed images obtained from the fabricated composites. Images observed from LF-M0 showed some particles that were thought to be impurities of the white cement. However, their planar occupation area was so small that it was negligible in a comparison study of the $q$ value.

It is generally agreed that an increase in the MWNT content is accompanied with an increase in the total area of MWNT if it is uniformly distributed throughout composites. Such phenomena was in close agreement with the test result provided in Fig. 7 where the total area of MWNT was observed to increase with the MWNT content. In addition, the MWNT clumps were not found even when the MWNT content was increased up to $1.5 \%$. Observation of the images thus indicated that MWNT was satisfactorily distributed throughout the composites in the LF-M group.
In an effort to express the change of MWNT distribution states in a quantitative manner, the $q$ value was calculated for the LF-M group, as shown in Fig. 8. The $q$ values of the group exhibited a steady increase as a function of the MWNT content in the composites. Accordingly, a dramatic increase in the electrical conductivity is expected as the MWNT content increases.

An image analysis of the MWNT distribution state in the composites fabricated under the high flow condition was not carried out because it was not possible to present reliable $q$ values due to the presence of the MWNT clumps, as shown in the W42-M06 specimen of the Fig. 3.

\subsubsection{Conductivity of the Composites Incorporating Various MWNT Contents}

The change of the DC conductivity of composites fabricated under the two different flow condition, the low and high flow conditions, with various MWNT contents was investigated here. The constituent materials and respective weight content ratios of the composites fabricated under the low flow condition are given in the Table 2. The DC conductivity of the composites in the LF-M group is shown as a function of the MWNT content in Fig. 9. DC conductivity of $1.7 \mathrm{~S} \mathrm{~m}^{-1}$ was attained for specimen LF-M1.5. This value was greater than the electrical conductivity of $5 \mathrm{~mm}$ long

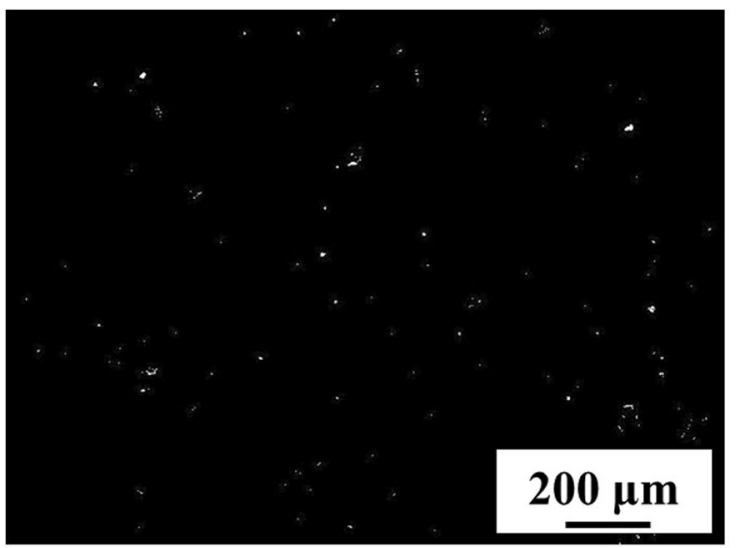

(a) LF-M0

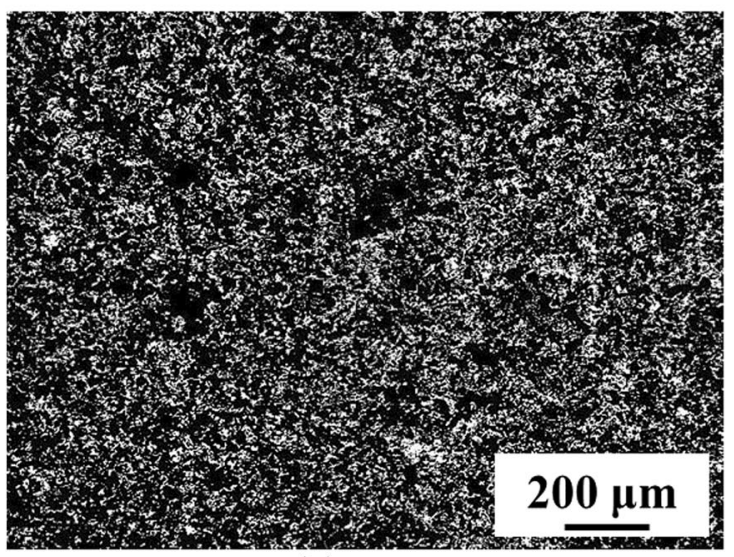

(c) LF-M0.6

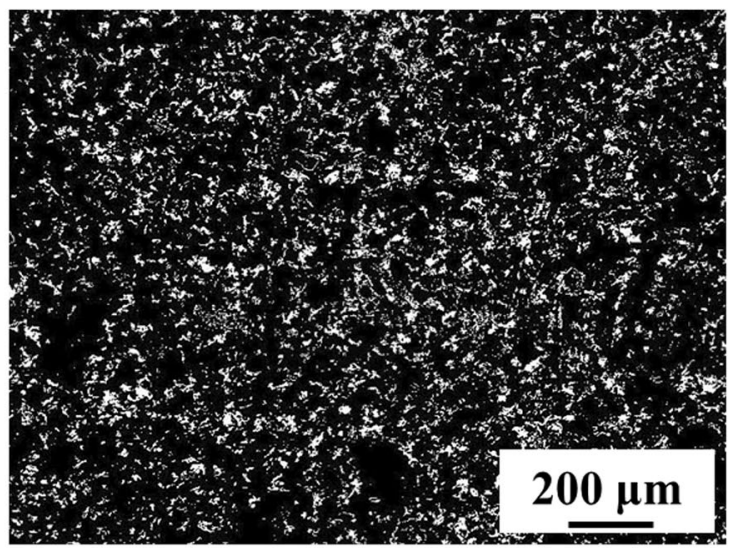

(b) LF-M0.3

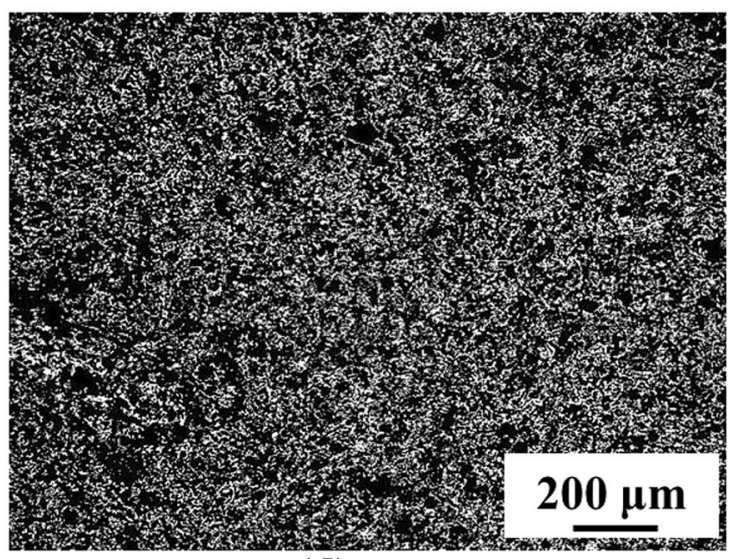

(d) LF-M1.5

Fig. 7 The fractured surface of the cement composites of the LF-M group fabricated under the low flow condition after having image thresholding, reversion, and binarization processes (Image size: $570 \times 426$ pixel): a LF-M0, b LF-M0.3, c LF-M0.6, and $\mathbf{d}$ LF-M1.5. 


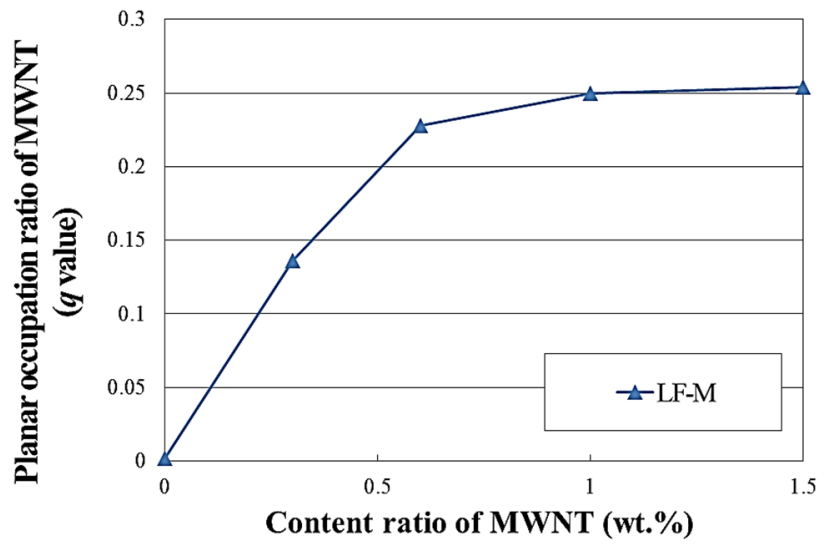

Fig. 8 A comparison of the $q$ value, which is the proportion of MWNT agglomerates to total area of the image, of the specimens in the LF-M group.

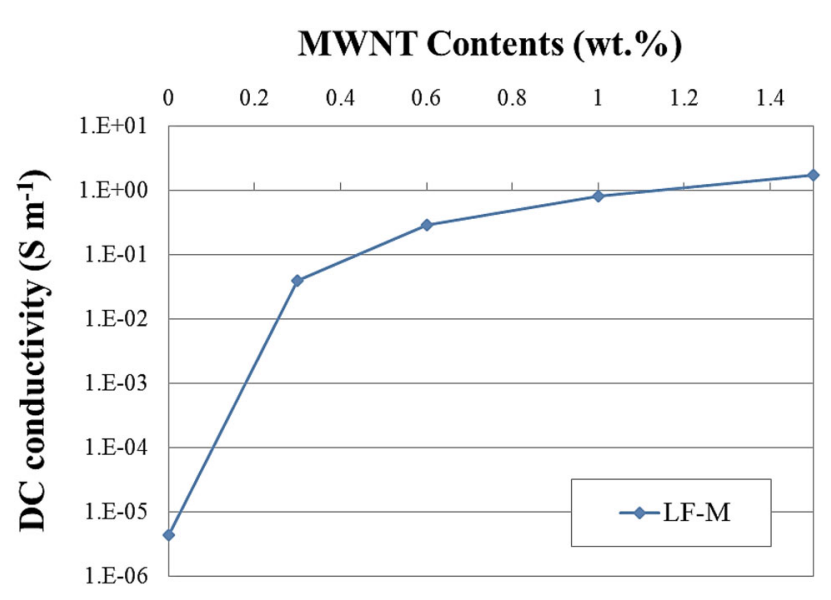

Fig. 9 The experimental conductivity values of the specimens fabricated under the low flow condition $(114 \mathrm{~mm}<$ flow $<126 \mathrm{~mm})$.

carbon fiber-incorporated cement composites and carbon black-filled cement composites (Wen and Chung 2007; Li et al. 2006). The high DC conductivity of the composites in the present section and the image analysis conducted in Sect. 4.2.1 indicated that MWNT was well distributed in the cement matrix.

The electrical percolation phenomenon of the LF-M group was found in the Fig. 9. The percolation threshold, which refers to the critical volume fraction of MWNT inducing remarkable change in the conductive phase, existed in a MWNT content range of $0-0.3 \mathrm{wt} \%$ for the LF-M group. Accordingly, the percolation threshold corresponded to $0.196 \mathrm{vol} \%$ if it is determined as the mean value of the percolation threshold range.

The percolation phenomena manifested in the MWNTembedded cement composites fabricated under the low flow condition indicated that MWNT was well distributed throughout the composites and the MWNT distribution was consistent in the specimen group. The results supported the image analysis results obtained in Sect. 4.2.1. Based on the image analysis results and the electrical percolation

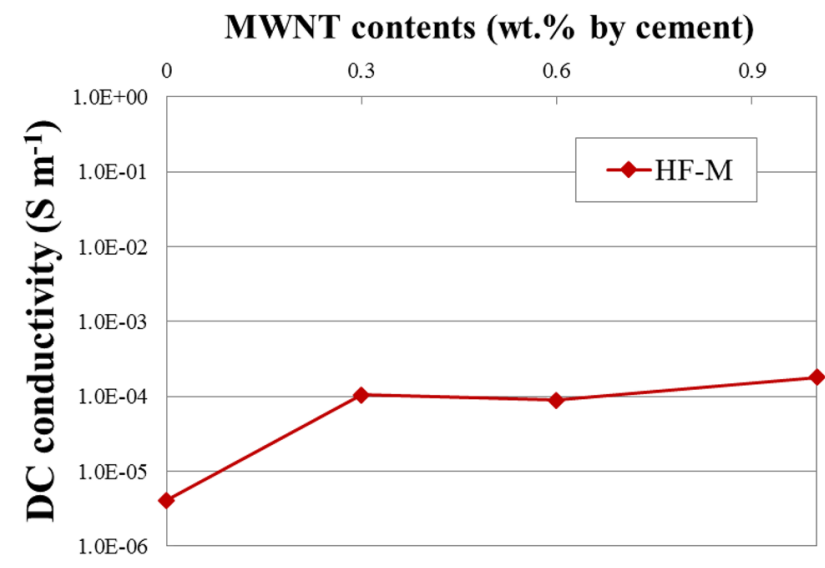

Fig. 10 The variations of the DC conductivity of the specimens fabricated under the high flow condition (flow $>250 \mathrm{~mm}$ ) are plotted as a function of the MWNT content.

phenomena, an acceptable MWNT distribution was attained by reducing the flow of the composites. Consequently, it can be concluded that maintaining the low flow of fresh mixtures of the composites is crucial to improve the MWNT distribution.

The change of the DC conductivity of the composites fabricated under the high flow condition with various MWNT contents was also investigated. The constituent materials and respective weight content ratios of the composites are given in Table 4 (Nam et al. 2012). MWNT was incorporated in each composite type at $0,0.3,0.6$, and $1.0 \mathrm{wt} \%$ by weight of cement. To fabricate specimens with high flow, the $\mathrm{W} / \mathrm{C}$ ratio was fixed to 0.4 in the composites and the SP/C ratio was 0.016 .

Figure 10 presents the change of the DC conductivity of the composites as a function of the MWNT content. The electrical percolation phenomenon was not found in Fig. 10. The DC conductivity of all the composites did not exceed $0.001 \mathrm{~S} \mathrm{~m}^{-1}$, hence all the composites appeared to be under the percolation threshold. The under percolation behavior of the DC conductivity was attributed to a poor distribution of MWNT in the composites. In addition, the DC conductivity of the composites may be dependent on many factors such as the dry condition of the specimens, void ratio, contact resistance between electrodes and the composite specimens, etc. In conclusion, it is not recommended that MWNT-embedded cement composites are fabricated under a high flow condition unless appropriate dispersion methods were carried out.

\section{Conclusion}

In the present work, the MWNT distribution in MWNTembedded cement composites was evaluated by a newly proposed image analysis method as well as electrical conductivity measurement. In addition, the influence of the fresh mixture's flow on the MWNT distribution in the composites was experimentally studied on the basis of the two evaluation methods. The conclusions derived from the present work can be summarized as follow. 
(1) The MWNT distribution, which was evaluated by the planar occupation ratio of MWNT, $q$, was enhanced as the flow of fresh composite mixtures decreased.

(2) The DC conductivity of MWNT-embedded cement composites fabricated with different flows was examined and it was observed that the conductivity increased as the flow was decreased.

(3) The image analysis of the MWNT-embedded cement composites fabricated under the low flow condition $(114 \mathrm{~mm}<$ flow $<126 \mathrm{~mm})$ revealed that the $q$ value linearly increased as the MWNT content increased. The linear relationship between the $q$ value and the MWNT content demonstrated that fabrication of the composites under the low flow condition is an effective way to enhance the MWNT distribution.

(4) The DC conductivity of the MWNT-embedded cement composites fabricated under the low flow condition showed percolation phenomena, thus indicating that MWNT was well distributed in the composites, as evaluated in the image analysis.

The proposed image analysis procedures for evaluation of the MWNT distribution can be used as an evaluation method to quantify the distribution of carbon nano-materials in the cement matrix. The conductive MWNT-embedded cement composites with percolative networks produced by controlling the flow are expected to be utilized as piezoresistive sensors, EMI shielding materials, electrostatic discharge materials, heating elements, etc. Future work will be focused on in-depth study of the origin of the flow effect on the MWNT distribution.

\section{Acknowledgments}

This research was supported by the KUSTAR-KAIST Institute, KAIST, Korea and also sponsored by a research project 'Development of fabrication method for floor heating material by use of CNT-cement composites with self-heating capacity of $5 \mathrm{~W}-50^{\circ} \mathrm{C}^{\prime}$ (Project code: 15CTAP-C098086-01) funded by Ministry of Land, Infrastructure and Transport (MOLIT) of Korea Government and Korea Agency for Infrastructure Technology Advancement (KAIA). Authors also deeply thank Prof. Hyeong-Ki Kim in Chosun University and Prof. SungMin Choi in KAIST for their important comments on this research and acknowledge June Lee in National NanoFab Center for cooperation in the image analysis.

\section{Open Access}

This article is distributed under the terms of the Creative Commons Attribution 4.0 International License (http:// creativecommons.org/licenses/by/4.0/), which permits unrestricted use, distribution, and reproduction in any medium, provided you give appropriate credit to the original author(s) and the source, provide a link to the Creative Commons license, and indicate if changes were made.

\section{References}

ASTM International. (2013). ASTM C1437-standard test method for flow of hydraulic cement mortar. West Conshohocken, PA: ASTM.

Daimon, M., \& Roy, D. M. (1979). Rheological properties of cement mixes: II. Zeta potential and preliminary viscosity studies. Cement and Concrete Research, 9(1), 103-109.

Fan, M. Z., Bonfield, P. W., Dinwoodie, J. M., \& Breese, M. C. (2000). Dimensional instability of cement bonded particleboard: SEM and image analysis. Journal of Materials Science, 35(24), 6213-6220.

Kang, S. T., Lee, B. Y., Kim, J. K., \& Kim, Y. Y. (2011). The effect of fibre distribution characteristics on the flexural strength of steel fibre-reinforced ultra high strength concrete. Construction and Building Materials, 25(5), 2450-2457.

Kim, H. K., Nam, I. W., \& Lee, H. K. (2014). Enhanced effect of carbon nanotube on mechanical and electrical properties of cement composites by incorporation of silica fume. Composite Structures, 107, 60-69.

Konsta-Gdoutos, M. S., Metaxa, Z. S., \& Shah, S. P. (2010). Highly dispersed carbon nanotube reinforced cement based materials. Cement and Concrete Research, 40(7), 1052-1059.

Lee, B. Y., Kim, J. K., Kim, J. S., \& Kim, Y. Y. (2009). Quantitative evaluation technique of polyvinyl alcohol (PVA) fiber dispersion in engineered cementitious composites. Cement and Concrete Composites, 31(6), 408-417.

Lee, Y. H., Lee, S. W., Youn, J. R., Chung, K., \& Kang, T. J. (2002). Characterization of fiber orientation in short fiber reinforced composites with an image processing technique. Materials Research Innovations, 6, 65-72.

Li, J., Ma, P. C., Chow, W. S., To, C. K., Tang, B. Z., \& Kim, J. K. (2007). Correlations between percolation threshold, dispersion state, and aspect ratio of carbon nanotubes. Advanced Functional Materials, 17(16), 3207-3215.

Li, H., Xiao, H., \& Ou, J. (2006). Effect of compressive strain on electrical resistivity of carbon black-filled cement-based composites. Cement \& Concrete Composites, 28(9), 824-828.

Liu, J., Li, C., Liu, J., Du, Z., \& Cui, G. (2011). Characterization of fiber distribution in steel fiber reinforced cementitious composites with low water-binder ratio. Indian Journal of Engineering and Materials Sciences, 18, 449-457.

Nam, I. W., Kim, H. K., \& Lee, H. K. (2012). Influence of silica fume additions on electromagnetic interference shielding effectiveness of multi-walled carbon nanotube/cement composites. Construction and Building Materials, 30, $480-487$.

Nam, I. W., Souri, H., Lee, H. K. (2015). Percolation threshold and piezoresistive response of multi-wall carbon nanotube/ cement composites. Smart Structures and Systems (in review).

Otsu, N. (1979). A threshold selection method from gray-level histograms. IEEE Transactions on Systems, Man, and Cybernetics, 9(1), 62-66. 
Ozyurt, N., Woo, L. Y., Mason, T. O., \& Shah, S. P. (2006). Monitoring fiber dispersion in fiber-reinforced cementitious materials: Comparison of AC-Impedance Spectroscopy and Image Analysis. ACI Materials Journal, 103(5), 340-347.

Redon, C., Chermant, L., Chermant, J. L., \& Coster, M. (1999). Automatic image analysis and morphology of fibre reinforced concrete. Cement \& Concrete Composites, 21(5-6), 403-412.

SEMI MF 43 (2005) Standard test methods for resistivity of semiconductor materials.

Sorensen, C., Berge, E., \& Nikolaisen, E. B. (2014). Investigation of fiber distribution in concrete batches discharged from ready-mix truck. International Journal of Concrete Structures and Materials, 8(4), 279-287.

Stauffer, D., \& Aharony, A. (1994). Introduction to percolation theory Revised (2nd ed.). London, UK: Taylor \& Francis.
Striolo, A., Chialvo, A. A., Gubbins, K. E., \& Cummings, P. T. (2005). Water in carbon nanotubes: Adsorption isotherms and thermodynamic properties from molecular simulation. The Journal of Chemical Physics, 122(23), 234712.

Vance, K., Aguayo, M., Dakhane, A., Ravikumar, D., Jain, J., \& Neithalath, N. (2014). Microstructural, mechanical, and durability related similarities in concretes based on OPC and alkali-activated slag binders. International Journal of Concrete Structures and Materials, 8(4), 289-299.

Wen, S., \& Chung, D. D. L. (2007). Double percolation in the electrical conduction in carbon fiber reinforced cementbased materials. Carbon, 45(2), 263-267.

Xie, P., Gu, P., \& Beaudoin, J. J. (1996). Electrical percolation phenomena in cement composites containing conductive fibres. Journal of Materials Science, 31(15), 4093-4097. 